\title{
A comparison of cytokine production in 2-dimensional and 3-dimensional cultures of bone marrow stromal cells of muliple myeloma patients in response to RPMI8226 myeloma cells
}

\author{
Barbara Zdzisińska1 ${ }^{1}$ Jacek Roliński², Tomasz Piersiak² \\ Martyna Kandefer-Szerszeń ${ }^{1}$
}

\author{
${ }^{1}$ Department of Virology and Immunology, Maria Curie-Skłodowska University, Lublin, Poland \\ 2Department of Clinical Immunology, Medical University, Lublin, Poland \\ ${ }^{3}$ Department of Comparative Anatomy and Anthropology, Maria Curie-Skłodowska University, Lublin, \\ Poland
}

\begin{abstract}
We examined cytokine production by bone marrow stromal cells (BMSCs) of patients with multiple myeloma (MM) in response to contact with myeloma RPMI8226 cells in standard 2-dimensional (2D) cultures and in 3-dimensional (3D) cultures on a gelatine sponge scaffold. It was detected that BMSCs in the 3D cultures produced more IL-11 and HGF and less IL-10 than in the 2D cultures. Moreover, RPMI8226 cells after contact with BMSCs in 3D cultures produced more sIL-6R than in the classic 2D cultures. We concluded that 3D cultures of BMSCs with myeloma cells offered a promising model for in vitro examination of interactions between myeloma cells and the bone marrow stroma and for examination of potent antimyeloma agents.
\end{abstract}

Keywords: 3 -dimensional, cytokines, multiple myeloma, bone marrow stromal cells

\section{Introduction}

Multiple myeloma (MM) is characterized by monoclonal proliferation of malignant plasma cells that infiltrate the bone, initiating osteoclast activity and extensive osteolysis. The bone marrow environment, which is conducive to myeloma cell growth and survival, consists of several cell types intimately involved in the evolution and propagation of myeloma bone lesions, which include stromal cells, osteoblasts, bone marrow leukocytes, endothelial cells and osteoclasts. These cells communicate through a complex series of molecular pathways including adhesion molecules, cytokines, chemokines, growth factors and their respective receptors that may be critical for disease progression. Previous studies suggest that bone marrow stromal cells (BMSCs) in myeloma patients differ from those of healthy donors $[1-4,8,19,20]$. We report-

Correspondence: B. Zdzisińska, Dept. of Virology and Immunology, Maria Curie-Skłodowska University, Akademicka Str. 19, 20-033 Lublin, Poland; tel.: (+4881) 5375936, fax.: (+4881) 5375959, e-mail: basiaz@biotop.umcs.lublin.pl ed elsewhere that BMSCs of multiple myeloma patients, in comparison to a control, produced more IL-6, IL-10, TNF- $\alpha$, HGF, OPN and BAFF in response to RPMI8226 cells [21].

All of those experiments, concerning the abnormal synthesis of different cytokines and elements of the extracellular matrix and the abnormal expression of genes involved in angiogenesis, osteogenic differentiation or tumor growth, were done on BMCSs cultivated in two-dimensional (2D) conditions. It has been detected, however, that 2D culture systems do not allow one to fully explore the interactions between the cells of bone marrow because such cultures fail to reproduce several critical aspects of the three-dimensional (3D) structure of the bone marrow microenvironment, including its high cell density and the microanatomical relationships between cells. For these reasons, a 3D culture system provides an attractive model to examine normal and abnormal cell interactions in the marrow. 3D BMSC cultures have already been used to examine age-related changes in the production of cytokines, such as IL-6 or osteoprotegerin (OPG) $[11,13]$. 
In this report, we describe the influence of the cell culture model on the production of several cytokines, previously detected to be differentially produced by BMSCs of MM patients and healthy controls [21]. We measured cytokine production in myeloma patients' BMSCs cultured on plastic plates (2D cultures) and in gelatine sponge discs (3D cultures) resembling the organic component of bones.

\section{Materials and methods}

Patients. Bone marrow samples from 16 patients with newly diagnosed MM were obtained with the patients ' written informed consent. The patients were 8 males and 8 females. The disease stage according to Durie and Salmon [6] was estimated, and four patients were at stage I, five at stage II, and seven at stage III of the disease. The study was approved by the Local Ethical Committee. The characteristics of the subjects are shown in Table 1.

Bone marrow stromal cells. Bone marrow from the patients and controls was obtained by sternal puncture. Anti-coagulated bone marrow samples were diluted 1:2 with Iscove's Modified Dulbecco`s Medium (IMDM; Gibco, BRL, UK) containing $0.2 \%$ methylcellulose (Sigma, St. Louis, MO, USA). After sedimentation of erythrocytes (30-40 $\mathrm{min})$ at room temperature, supernatants were collected, washed twice with IMDM supplemented with $2 \%$ fetal bovine serum (FBS; Gibco), and resuspended in IMDM supplemented with $10 \%$ FBS, $10 \%$ horse serum (Gibco), $1 \mu \mathrm{M}$ hydrocortisone (Sigma) and 1\% antibiotic-antimycotic solution (Gibco). Cells from the supernatants were seeded in T25 cell culture flasks (Nunc, Roskilde, Denmark) at a density of $2 \times 10^{6} \mathrm{cell} / \mathrm{ml}$. The cultures were incubated for 4 weeks at $37^{\circ} \mathrm{C}$ in a humidified atmosphere containing $5 \% \mathrm{CO}_{2}$. After 1,2 and 3 weeks of incubation all non-adherent cells were removed together with the medium. After 4 weeks of incubation, a continuous network of adherent cells occupying the entire bottom of each flask was obtained, and adherent cells were treated with $0.25 \%$ trypsin (Sigma) and frozen in IMDM medium supplemented with 20\% FBS and $10 \%$ DMSO in liquid nitrogen until use in the experiments. After thawing, the cells were propagated in T75 flasks in IMDM medium supplemented with $10 \%$ FBS. Characterization of BMSCs by flow cytometry revealed that $75.6 \pm 11.9 \%$ of cells were CD166 positive, CD166 being the marker for human bone marrow stroma fibroblasts [17]. $13.1 \pm 6.0 \%$ cells were $\mathrm{CD} 14$ positive, indicating the presence of monocytes and macrophages. No CD38+/CD138+ plasma cells were detected in any of the BMSC cultures.

Cytokine induction. After trypsinization, BMSCs were suspended in IMDM medium with $10 \%$ FBS and seeded at a density of $2 \times 10^{5}$ cell/ml into 24-well plastic plates (Nunc) $(1 \mathrm{ml} /$ well). After $24 \mathrm{~h}$ of incubation, supernatants were removed, and new IMDM medium supplemented with $2 \%$ FBS was added. The cultures were divided into two batches. The first batch of cultures consisted of BMSCs seeded on 6-well plastic plates and served as a control of spontaneous cytokine production by BMSCs. Into the second batch of BMSCs, myeloma RPMI8226 cells (ECACC 87012702) were added, at a density of $1 \times 10^{6} \mathrm{cell} / \mathrm{ml}$ in IMDM medium supplemented with $2 \%$ FBS (direct cell-to-cell contact). The cultures were incubated for $24 \mathrm{~h}$ or $72 \mathrm{~h}$ at $37^{\circ} \mathrm{C}$, and cytokine concentrations in supernatants were measured by ELISA.

Collagen discs, $5 \mathrm{~mm}$ diameter, $300-500 \mathrm{~m}$ pores (Spongostan $^{\mathrm{R}}$ Special), were seeded with BMSCs at a density of $2 \times 10^{5}$ cells/disc in $1 \mathrm{ml}$ of IMDM medium supplemented with $2 \%$ FBS and incubated for $24 \mathrm{~h}$ at $37^{\circ} \mathrm{C}$ in siliconized tubes in a gyratory water bath $(100 \mathrm{rpm})$ in an incubator with the flow of air supplemented with
$5 \% \mathrm{CO}_{2}$. After that, the medium was changed to a new one, and into some tubes $1 \times 106$ RPMI8226 cells in $1 \mathrm{ml}$ of IMDM medium supplemented with $2 \%$ FBS were added. Supernatants were collected from cultures of BMSCs and co-cultures with myeloma cells after 24 or $72 \mathrm{~h}$ of incubation and assayed for cytokines by ELISA.

Assay for cytokines. The concentrations of IL-6, IL-10, IL-11 and HGF were measured by ELISA using commercially available kits from R\&D Systems, Minneapolis, MN, USA. The detection limit was $0.7 \mathrm{pg} / \mathrm{ml}$ for IL-6, $3.9 \mathrm{pg} / \mathrm{ml}$ for IL-10, $8.0 \mathrm{pg} / \mathrm{ml}$ for IL-11 and $40.0 \mathrm{pg} / \mathrm{ml}$ for HGF. The concentration of sIL-6R was measured by ELISA kit obtained from Bender MedSystems GmbH, Vienna, Austria. The detection limit was $0.01 \mathrm{ng} / \mathrm{ml}$.

Statistical analysis. Results were reported as mean \pm S.D. The two-way ANOVA test with post-hoc Tukey`s test was used. The concentrations of cytokines below the detection level were considered as 0 for the purpose of the analysis. P values of 0.05 or less were considered significant.

\section{Results and discussion}

As can be seen from Fig 1., BMSCs seeded at a density of $2 \times 10^{5} \mathrm{cell} / \mathrm{s} / \mathrm{disc}$ on gelatine foam, attached to the pore surface and, after $24 \mathrm{~h}$ of incubation, covered nearly all the entire surface of the gelatine sponge. At that stage RPMI8226 cells at a density of $1 \times 10^{6}$ cells/ml (ratio 1:5) were added and cultivated for additional $24 \mathrm{~h}$ or $72 \mathrm{~h}$. The level of several cytokines was measured in the supernatants. As can be seen from Fig. $2 \mathrm{~A}$, the level of IL-6 produced spontaneously, without induction, increased with the time of incubation and was similar in the 2D and the 3D cultures. The addition of RPMI8226 cells to BMSCs resulted in a significant drop in the IL-6 level. This phenomenon was probably caused by IL- 6 binding to its soluble receptor (sIL$6 \mathrm{R}$ ), as in co-cultures a significant increase of sIL-6R produced by RPMI8226 cells was observed (Fig. 2B). It seems likely that IL-6 bound to sIL-6R was no longer detectable by the monoclonal antibodies used in the ELISA test. On the other hand, we had already reported a significant increase of intracellular IL-6 produced by BMSCs of MM patients after contact with RPMI8226 myeloma cells when the IL-6 level was measured inside the cells by flow cytometry [21]. A measurement of sIL-6R revealed that it was mainly produced by myeloma RPMI 8826 cells, especially after contact with BMSCs. Moreover, in 3D cultures the sIL-6R concentration was significantly higher than in $2 \mathrm{D}$ cultures.

In contrast to sIL-6R, IL-10 was produced by BMSCs of MM patients nearly exclusively after cellto-cell contact with RPMI8226 cells (Fig. 2C). When we compared its production in 2D and 3D cultures, a significant decrease of IL-10 production was observed in the latter, indicating differences in the induction of signals generated in spatial interactions between of both types of cells, in comparison to the flat 2D cultures. 
Table 1. Clinical characteristics of multiple myeloma patients.

\begin{tabular}{|l|c|}
\hline \multicolumn{1}{|c|}{ Feature } & $\begin{array}{c}\text { MM } \\
\mathrm{n}=16\end{array}$ \\
\hline$\Lambda$ ge (mean ) & 63.6 \\
\hline$\Lambda$ ge (range) & $49-83$ \\
\hline Men / women (n) & $8 / 8$ \\
\hline $\begin{array}{l}\text { Disease stage acc. to Durie and Salmon }[9] \\
\mathrm{I} / \mathrm{Il} / \mathrm{Ill}\end{array}$ & $4 / 5 / 7$ \\
\hline$\%$ of plasma cells in bone marrow (mean $\pm \mathrm{SD})$ & $25.45 \pm 17.74$ \\
\hline Ostcolysis 0/1/2/3 & $3 / 1 / 3 / 9$ \\
\hline
\end{tabular}

In the 2D model, co-culture of BMSCs with RPMI8226 cells did not increase HGF production, but in the 3D model, HGF production in co-cultures of both types of cells was significantly increased (Fig. 2D). RPMI8226 cells alone produced a level of HGF below the detection limit of the ELISA kit, and these results were not considered.

Similarly to HGF, a significant increase in IL-11 production in 3D co-cultures of BMSCs with RPMI8226 cells, in comparison to the 2D model of coculture, was observed (Fig. 2E).

We believe that 3D system reproduces the conditions of the bone marrow environment better than do 2D cultures and can be useful in examining the interactions of bone marrow stromal cells with the extracellular matrix (collagen) and with other cells, such as myeloma cells.

Our studies showed that the level of cytokine expression was dependent on the conditions of culture of BMSCs and their co-culture with RPMI8226 cells. In the 3D model, in which the gelatine sponge mimicked several aspects of the bone marrow microenvironment, including the organic component of bone, its architecture, high cell density and intimate interactions between stromal cells and myeloma cells, the expression of IL-11, sIL-6R and HGF in BMSCs in cell-to-cell contact with myeloma cells was significantly higher than in 2D cultures. In contrast to that, IL-10 production was significantly lower. IL-11 and IL-6 have both been reported to stimulate growth, promote osteoclastogenesis and bone resorption in bone marrow cultures $[9,10]$. Recent studies have identified HGF as a potential key signal in the evolution of myeloma, as HGF exerts a strong antiproliferative and antiapoptotic effect on myeloma cells [18]. Therefore, overexpression of IL-11, HGF and sIL-6R, and decreased production of IL-10 seem to favour overgrowth of myeloma cells in the bone marrow microenvironment. Importantly, cell-to-cell contact of BMSCs with RPMI8226 cells did not induce IL-11 and HGF production in $2 \mathrm{D}$ cultures, unlike in the $3 \mathrm{D}$ model where it was a strong signal for overproduction of IL-11 and
HGF. Also, production of sIL-6R, which is known to enhance the activity of IL-6 [12] was significantly enhanced in the 3D model of multiple myeloma. These results strongly indicate that combination of $3 \mathrm{D}$ culture of BMSCs using gelatine sponge as a scaffold with coculture with myeloma cells offers a promising model for in vitro examination of interactions between cells which occur in vivo in patients with multiple myeloma.

Spongostan ${ }^{\mathrm{R}}$ gelatine sponge has been shown to be a useful cell carrier for different purposes. For example, it was used to cultivate human osteoblasts derived from bone marrow stem cells in a bioreactor for application in the alveoloplasty of cleft palate [16]. This scaffold, as the authors explain, was chosen because of its availability, biocompatibility, reabsorbability, osteoinductivity and absence of allergic reactions or other side effects in patients. Spongostan has also been used as a scaffold for chemosensitivity examination of malignant astrocytic tumors isolated from patients. Patients were treated with those cytostatics which exhibited a high growth inhibitory rate in 3-dimensional cultures. Individual chemotherapy based on in vitro chemosensitivity contributed to longer survival of the treated patients [14].

It has already been detected that cultures of human bone marrow cells cultivated on 3D scaffolds in comparison to those cultivated in a 2D monolayer exhibit a significantly different response to the toxicity of trimethoprim and pyrimethamine [5].

Growth in 3D architecture has been shown to promote resistance of cancers to treatment with drugs, cytokines, or irradiation, thereby potentially playing an important role in tumor expansion. Studies revealed that $3 \mathrm{D}$ cultures had an overall impact on gene expression in melanoma HBL and NA8 cell lines. Cells cultivated as multicellular tumor spheroids exhibited increased expression of genes encoding proangiogenic and melanoma growth factors as compared to standard 2D cultures [7].

CD34+ cord blood cells cultivated in 3D cultures containing a collagen I matrix exhibited quantitative changes in gene expression. Gene expression analysis with the microarray chip technology revealed an upregulation of more than 50 genes in 3D cultures in the presence of collagen I. Among these, genes for several growth factors, cytokines and chemokines were overexpressed. For example IL-8, IL-1, IL-6, and HGF gene expression was enhanced [15].

It was detected in our experiments that BMSCs in the $3 \mathrm{D}$ cultures produced more IL-11 and HGF and less IL-10 than in the 2D cultures. The results of our study strongly indicate that combination of 3D culture of BMSCs using gelatine sponge as a scaffold with co culture with myeloma cells offers a promising model for in vitro examination of interactions between the cells which occur in vivo in patients with multiple myeloma in the specific bone marrow microenviron- 

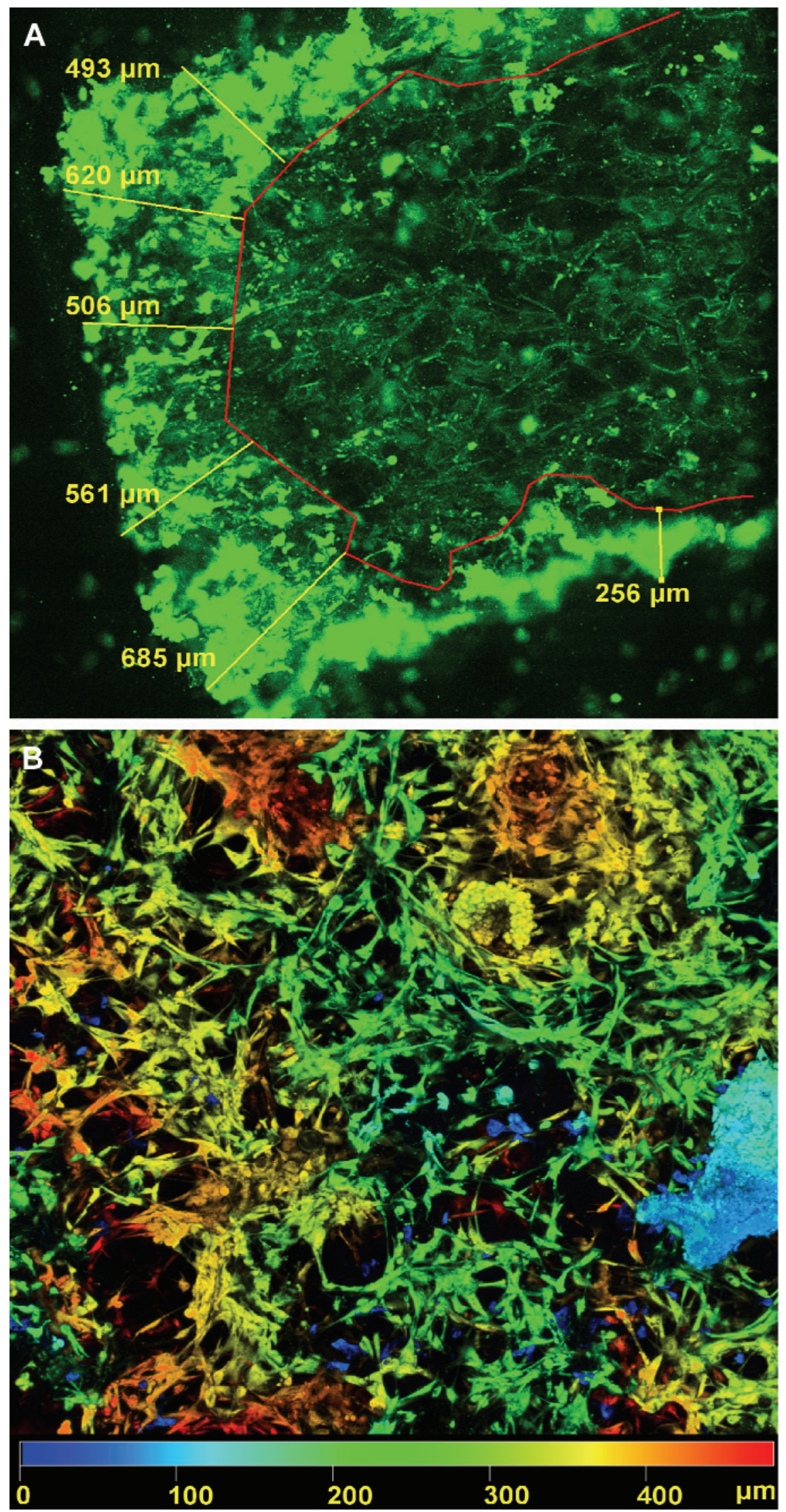

Fig. 1. Confocal Scanning Microscopy (LSM 5 PASCAL, Carl Zeiss, Germany) was used to obtain images of spongostan coated with BMSCs. All data were collected in fluorescent channel $488 \mathrm{~nm}\left(\lambda_{\mathrm{EX}}=\right.$ $\left.488 \mathrm{~nm}, \lambda_{\mathrm{EM}}=505 \mathrm{~nm}\right)$. BMSCs were prepared for confocal microscopy by staining the viable cells with the fluorescent dye 3,3' Dihexyloxacarbo-cyanine iodide (DIO3(6)). The 3D cultures were incubated with $0.5 \mu \mathrm{g} / \mathrm{ml}$ of DIO3(6) in complete media for $10 \mathrm{~min}$ (in the dark, at room temperature). Following a rinse in PBS (3X) and a replacement with fresh PBS, the cell cultures were examined using a confocal microscope. (A) cross-sectional view of a spongostan (original magnification $\times 50$ ). (B) 3D Depth Cod (Color Coded Depth Map) of surface of spongostan. Color corresponds to the depth from the sponge surface, with blue being closest to the surface and red being at $400 \mu \mathrm{m}$ from the surface (original magnification $\times 50$ ). 


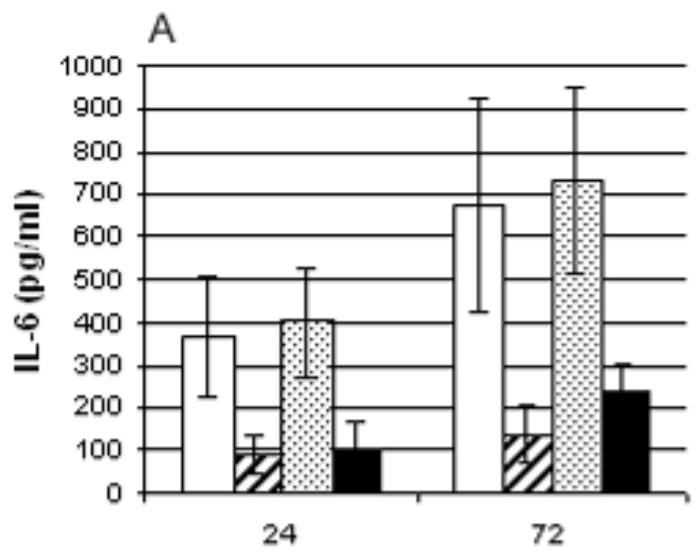

hours of incubation
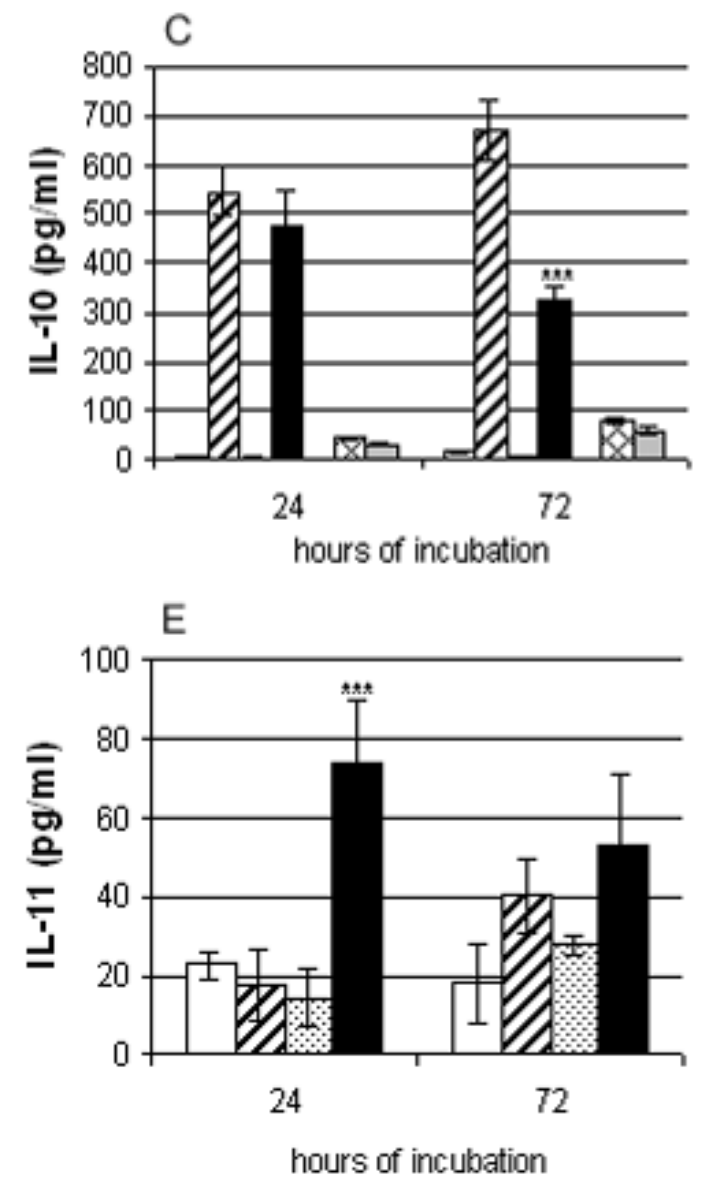

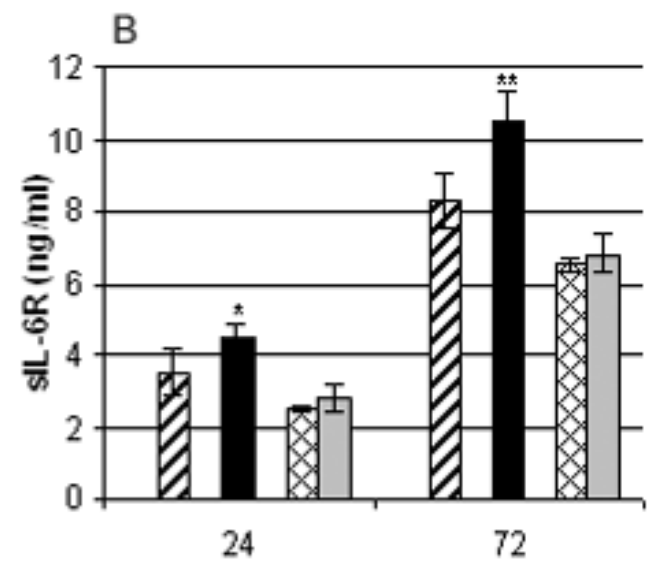

hours of incubation

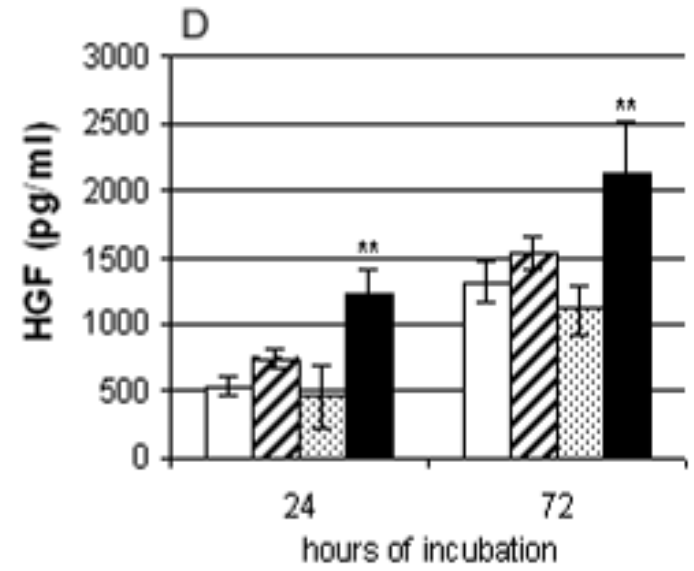

Fig. 2. Cytokine production was measured $24 \mathrm{~h}$ and $72 \mathrm{~h}$ after contact of BMSCs of MM patients with myeloma RPMI8226 cells. Each bar represents the average response of BMSCs of $16 \mathrm{MM}$ patients. BMSCs were cultivated in 2D or in 3D cultures. Bars: white, spontaneous production by BMSCs in 2D monocultures; striped, production in 2D cultures of BMSCs with RPMI8226 cells; spotted, production by BMSCs in 3D monocultures; black, production in 3D cultures of BMSC with RPMI 8226 cells; checked, production by RPMI8226 cells in 2D monocultures; grey, production by RPMI8226 cells in $3 \mathrm{D}$ monocultures. Values are mean \pm S.D. (the two-way ANOVA test with post-hoc Tukey`s test was used). ${ }^{*} \mathrm{p}<0.05 ;{ }^{* *} \mathrm{p}<0.01$; $* * * \mathrm{p}<0.001$ ment and a model for examination of potent antimyeloma agents.

Acknowledgment: This work was supported by a grant from Ministry of Science and Higher Education No 2 P05A 09528.

\section{References}

[ 1] Arnulf B, Lecourt S, Soulier J, Ternaux B, Lacassagne MN, Crinquette A, Dessoly J, Sciaini AK, Berbunan M, Chomienne C, Fermand JP, Marolleau JP, and Larghero J. Pheno- typic and functional characterization of bone marrow mesenchymal stem cells derived from patients with multiple myeloma. Leukemia. 2007;21:158-163.

[2] Calabrio A, Oken MM, Hascal VC, and Masellis AM. Characterization of hyaluronian synthetase expression and hyaluronian synthesis in bone marrow mesenchymal progenitor cells: predominant expression of HAS1 mRNA and up-regulated hyaluronian synthesis in bone marrow cells derived multiple myeloma patients. Blood. 2002;100:25782585.

[3] Caligaris-Cappio F, Bergui L, Gregoretti MG, Gaidano G, Gaboli M, Schena M, Zallone AZ and Marchisio PC. Role of 
bone marrow stromal cells in the growth of human multiple myeloma. Blood. 1991;77:2688-2693.

[4] Corre J, Mahtouk K, Attal M, Gadelorge M, Huynh A, FleuryCappellesco S, Danho C, Laharague P, Klein B, Reme T and Bourin P. Bone marrow mesenchymal cells are abnormal in multiple myeloma. Leukemia. 2007;21:1079-1088.

[5] Cui ZF, Xu X, Trainor N, Triffitt JT, Urban JP, Tirlapur UK. Application of multiple parallel perfused microbioreactors and three-dimensional stem cell culture for toxicity testing. Toxicol In vitro. 2007;21:1318-1324.

[6] Durie BG, Salmon SE. A clinical staging system for multiple myeloma. Correlation of measured myeloma cell mass with presenting clinical features, response to treatment and survival. Cancer. 1975;36:842-845.

[7] Ghosh S, Rosenthal R, Zjac P, Weber WP, Oertli D, Heberer M, Martin I, Spagnoli GC, Reschner A. Culture of melanoma cells in 3-dimensional architectures results in impaired immunorecognition by cytotoxic T lymphocytes specific for Malan-A/Mart-1 tumor- associated antigen. Ann Surg. 2005;242:851-858.

[8] Gregoretti MG, Gottardi D, Ghia P, Bergui L, Merico F and Marchisio PC. Characterization of bone marrow stromal cells from multiple myeloma. Leukemia Res. 1994;18:675-682.

[9] Ishikawa H, Tsuyama N, Obata M, and Kawano MM. Mitogenic signals initiated via inteleukin-6 receptor complexes in corporation with other transmembrane molecules in myelomas. J Clin Exp Hematopathol. 2006;46:55-66.

[10] Kudo O, Sabokbar A, Pocock A, Itonaga I, Fujikawa Y and Athanasou NA. Inteleukin-6 and IL-11 support human osteoclast formation by RANKL-independent mechanism. Bone. 2003;32:1-7.

[11] Makhluf HA, Mueller SM., Mizuno S, Glowacki J. Age-related decline in osteoprotegerin expression by human bone marrow cells cultured in three-dimensional collagen sponges. Biochem Biophys Res Commun. 2000;268:669-672.

[12] Mizuguchi H, Mizuno H, Yasukawa K, Ishiguro T, Fukui K, Imanaka T, Takagi M. Enhanced signal transduction by a directly fused protein of IL-6 and its receptor. J Biosci Bioeng. 2001;91:299-304.

[13] Mueller SM, Glowacki J. Age-related decline in the osteogenic potential of human bone marrow cells cultured in tree-dimensional collagen sponges. J Cell Biochem. 2001;82: 583-590.

[14] Ono A, Kanno H, Hayashi A, Nishimura S, Kuyama Y, Sato H, Ito S, Shimizu N, Chang CC, Yamamaoto I, Sasaki T, Tanaka M. Collagen gel matrix assay as an in vitro chemosensitivity test for malignant astrocytic tumors. Int $J$ Clin Oncol. 2007;12:125-130.

[15] Oswald J, Steudel C, Salchert K, Joergensen B, Thide C, Ehninger G, Warner C, Bornhauser M. Gene-expressing profiling of CD34+ hematopoietic cells expanded in a collagen I matrix. Stem Cells. 2006;24:494-500.

[16] Paganelli C, Fontana P, Porta F, Majorana A, Pazzaglia UE, Sapelli PL. Indication on suitable scaffold as carrier of stem cells in the alveoloplasty of cleft palate. J Oral Rehabil. 2006; 33:625-629.

[17] Stewart K, Monk P, Walsh S, Jefferiss CM, Letchford J, Beresford JN. STRO-1, HOP-26 (CD63), CD49a and SB-10 (CD166) as markers of primitive human marrow stromal cells and their more differentiated progeny: a comparative investigation in vitro. Cell Tissue Res. 2003;313:281-290.

[18] Tjin EPM., Derksen PWB., Kataoka H, Spaargaren M, and Pals SY. Multiple myeloma cells catalyze hepatocyte growth factor (HGF) activation by secreting the serine protease HGFactivator. Blood. 2004;104:2172-2175.

[19] Vincent T, Jourdan M, Sy MS, Klein B, and Mechti N. Hyaluronic acid induces survival and proliferation of human myeloma cells through an interleukin-6-mediated pathway involving the phosphorylation of retinoblastoma protein. J Biol Chem. 2001;276:14728-14736.

[20] Wallace SR, Oken MM, Lunetta KL, Panoskaltsis-Mortari A and Masellis AM. Abnormalities of bone marrow mesenchymal cells in multiple myeloma patients. Cancer. 2001;1:12191230 .

[21] Zdzisińska B, Bojarska-Junak A, Dmoszyńska A, KandeferSzerszeń M. Abnormal cytokine production by bone marrow stromal cells of multiple myeloma patients in response to RPMI8226 cells. Arch Immunol Ther Exp. 2008;56:1-15.

Submitted: 27 April, 2008 Accepted after reviews: 6 June, 2008 